\title{
INFRARED EDGE RADIATION BEAMLINE AT ALADDIN
}

\author{
T. E. May, R. A. Bosch and R. L. Julian, Synchrotron Radiation Center, \\ University of Wisconsin-Madison, 3731 Schneider Dr., Stoughton, WI 53589
}

\begin{abstract}
An infrared beamline has been constructed at the 800 $\mathrm{MeV}$ electron storage ring, Aladdin. The beamline is located downstream of a short straight section, where it collects edge radiation produced by electrons exiting and entering the bending magnets at the ends of the straight section. Measurements at wavelengths of $1-5 \mu \mathrm{m}$ suggest that the edge radiation is brighter than standard synchrotron radiation. When used for Fourier transform infrared (FTIR) microspectroscopy, the signal-to-noise ratio is 20 times that measured with a glower source. Several applications of the beamline are described.
\end{abstract}

\section{INTRODUCTION}

Long-wavelength radiation produced at the edge of a bending magnet was observed at the electron storage ring, Aladdin, in 1993 [1]. At the operating ring energy of 800 $\mathrm{MeV}$, the visible light several meters downstream of a straight section forms an intense whitish spot on axis with angular extent of several mrad. The light distribution changes to that of ordinary synchrotron radiation as one moves horizontally off axis. Similar features are evident in photographs taken at the Daresbury Laboratory [2]. At a ring energy of $250 \mathrm{MeV}$, the visible spot on axis is less pronounced, while colored crescents (again with an angular extent of several mrad) are observed whose position and intensity depends upon the steering bumps introduced into the straight section. The bright spot on axis appears to be "edge radiation" produced at the edge of a bending magnet [3]. The crescents are apparently the result of small deflections in quadrupole magnets caused by steering windings and misalignments $[4,5,6]$.

The measured spatial distribution of edge radiation at a wavelength $\lambda$ of $1 \mu \mathrm{m}$ agrees with computations [7]. Using lenses to image the sources of $0.5 \mu \mathrm{m}$ radiation within a straight section, the most intense source was observed at the location of the downstream bending magnet edge [8]. Additional weaker sources were also observed, consistent with radiation produced in quadrupoles by small deflections (with deflection angle $\leq$ $2 / \gamma$, where $\gamma$ is the relativistic mass factor [6]). From these images, the beam dimensions at the locations of the quadrupoles and edge were obtained, yielding very good agreement with theoretical values for horizontal beam sizes of $\sim 400 \mu \mathrm{m} \approx \lambda \gamma / 2$. Reasonable agreement was also obtained for vertical beam sizes of $\sim 80 \mu \mathrm{m} \approx \lambda \gamma / 10$ [8].

At the suggestion of $\mathrm{O}$. V. Chubar, we investigated the possibility of using edge radiation for an infrared (IR) beamline at Aladdin. Computations indicated that an edge radiation source might provide a greater brightness than ordinary synchrotron radiation, with a smaller opening angle [7]. In an experimental comparison of IR edge radiation and ordinary synchrotron radiation, described in Section 2, edge radiation allowed a greater flux to be imaged through pinholes smaller than $100 \mu \mathrm{m}$ with $\mathrm{f} / 4$ optics. This experiment suggests that the edge radiation is brighter, so it was decided to use an edge radiation source for the Aladdin IR beamline. To avoid conflict with insertion devices on the long straight sections, the IR beamline was constructed downstream of a short straight section, whose length is $3 \mathrm{~m}$.

\section{THE ALADDIN IR BEAMLINE}

The IR beamline is located at Port 031, an on-axis port at the end of a short straight section. The entrance aperture is $30.5 \mathrm{~mm}$ horizontal x $22.3 \mathrm{~mm}$ vertical, located $1.4 \mathrm{~m}$ downstream of the entrance poleface of a $30^{\circ}$ bending magnet. Imaging of visible radiation shows that the edge radiation source is located $10 \mathrm{~cm}$ upstream of the poleface. The measured bending magnet fringe field is approximately a linear ramp extending $10.8 \mathrm{~cm}$ from the poleface. Thus, the source is located at the entrance of the bending magnet fringe field, where the electron is deflected from its straight-line trajectory through an angle exceeding $1 / \gamma$ in several $\mathrm{cm}$. The entrance aperture is,

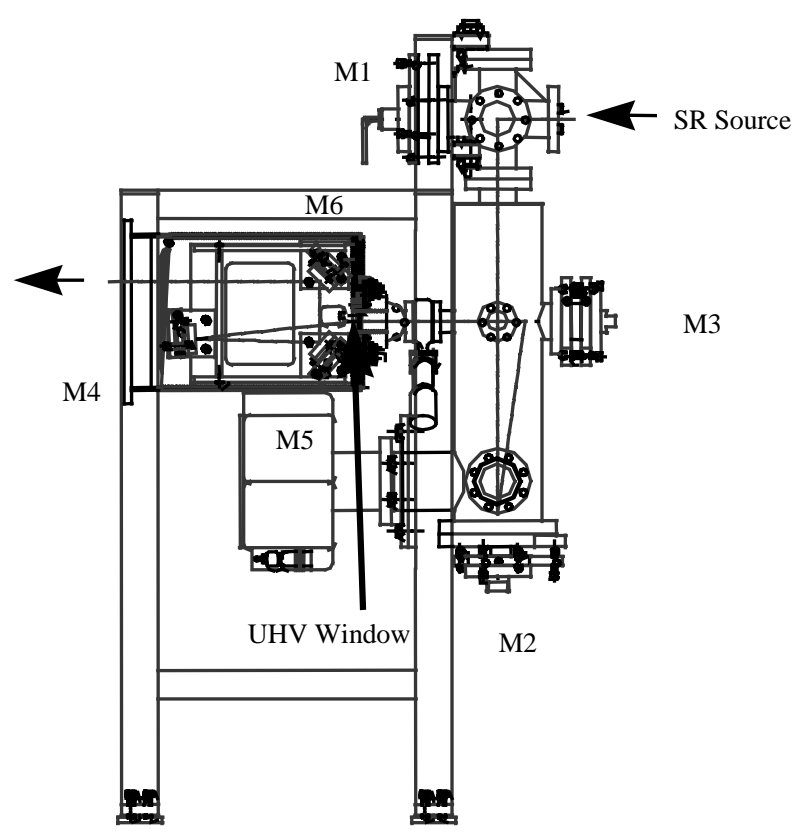

Figure 1. A diamond UHV window separates the two mirror boxes of the IR beamline. 
therefore, located $1.5 \mathrm{~m}$ downstream of the source, subtending $20 \mathrm{mrad}$ horizontal x $15 \mathrm{mrad}$ vertical.

Figure 1 shows the mirror boxes of the IR beamline. Each mirror box can be adjusted vertically and horizontally as a single unit to align to the port. Three mirrors in ultra-high vacuum (UHV) bring the IR light to a focus near its passage through a UHV window. Further downstream, three mirrors in rough vacuum collimate the light and transfer it to an FTIR interferometer. All mirrors are gold-coated; two are $\mathrm{f} / 6$ sphericals. The UHV window is type IIa diamond, $10 \mathrm{~mm}$ in diameter, $0.25 \mathrm{~mm}$ thick, with a $1.87^{\circ}$ wedge angle to prevent "channel" fringes. The beampipe is 2-inch stainless steel, terminated by a brass cell holding a $5-\mathrm{cm}$ diameter $\mathrm{KBr}$ window.

Downstream of the $\mathrm{KBr}$ window, a Nicolet Instrument Magna 550II FTIR bench with external source port is centered between two microscopes. Kinematic mounts support the FTIR bench and microscopes. The path length of the beam is modulated by the interferometer on the bench; the beam may be directed either to the sample compartment or to the side-port microscopes. A SpectraTech IR-Plan microscope is mounted on the right side of the FTIR bench. It has a $15 \mathrm{x}, 0.58$ numerical aperture (n.a.) Schwarzchild objective, 10x, 0.71 n.a. condenser, and an integral $0.25-\mathrm{mm}$ mercury cadmium telluride detector. The left side has a NicPlan microscope with computer-controlled sample stage and both $15 x$ and $32 x$ objectives. Apertures at focal planes upstream and downstream of the sample allow the beam to be restricted at the sample plane, permitting the highest spatial resolution.

At the operating ring energy of $800 \mathrm{MeV}$, FTIR measurements were performed to compare the edgeradiation source with a standard synchrotron radiation ("bend") source located in the uniform-field region of a bending magnet. For this comparison, standard synchrotron radiation was extracted through an aperture of size $28 \mathrm{~mm} \times 14 \mathrm{~mm}$ located $1.3 \mathrm{~m}$ downstream of the source, subtending $22 \mathrm{mrad}$ horizontal x $11 \mathrm{mrad}$ vertical. The edge radiation was extracted through an adjustable aperture $2.6 \mathrm{~m}$ downstream of the edge source, adjusted to

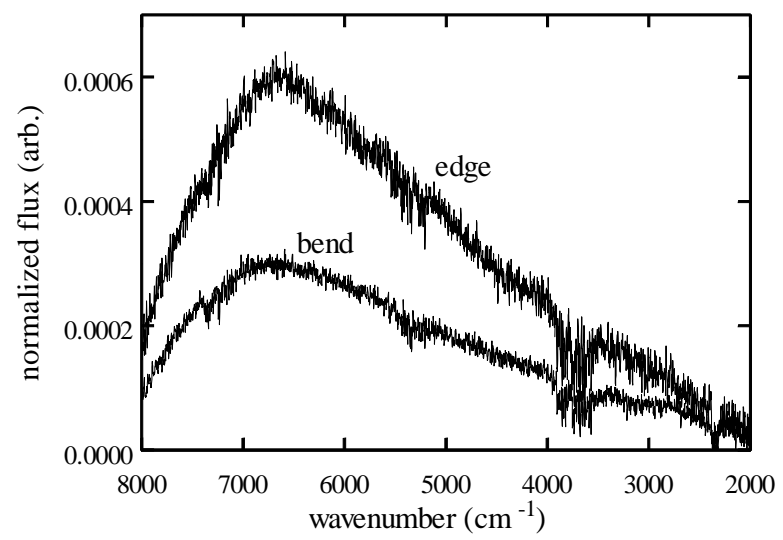

Figure 2. Flux focused through a 10 micron pinhole with $\mathrm{f} / 4$ optics, normalized to ring current.

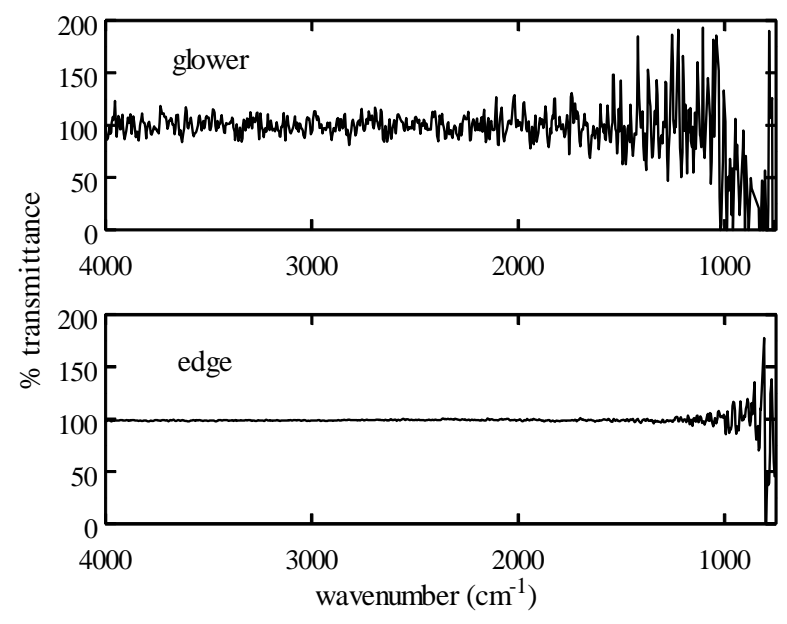

Figure 3. Transmittance data shows a 20 -fold noise reduction with edge radiation compared to a glower.

also subtend $22 \mathrm{mrad} \times 11 \mathrm{mrad}$. The bend measurements were limited to wavelengths below $5 \mu \mathrm{m}$ because a sapphire window was used. The microscopes were unavailable at the time, so a brightness comparison was performed using pinholes of diameter 400, 100, 25 and 10 $\mu \mathrm{m}$. The spot, focused in the FTIR sample compartment by an $\mathrm{f} / 4$ mirror, was $110 \mu \mathrm{m} \times 60 \mu \mathrm{m}$. A $400-\mu \mathrm{m}$ pinhole blocked most of the room-temperature blackbody radiation that peaks at $1000 \mathrm{~cm}^{-1}$.

The edge source produced less flux through the $400-\mu \mathrm{m}$ pinhole, nearly-equal flux through the $100-\mu \mathrm{m}$ pinhole, $70 \%$ more through $25-\mu \mathrm{m}$ pinhole, and $\sim 2$ times as much flux through the $10-\mu \mathrm{m}$ pinhole. Figure 2 shows currentnormalized edge and bend flux through a $10-\mu \mathrm{m}$ pinhole, collected for one minute at $4 \mathrm{~cm}^{-1}$ resolution. Because more flux is focused through small pinholes with the edge source, the data suggest that the edge source is brighter. Thus, it was decided to use the edge-radiation source for the Aladdin IR beamline.

Figure 2 also shows that the relative noise levels are about the same with the edge and bend sources. Because FTIR spectroscopy is sensitive to noise at $60 \mathrm{~Hz}$ and its harmonics, the choke for the bending magnets' power supply and several transformers were moved away from the electron orbit; the choke circuitry was also modified. These actions reduced the electron beam motion, improving the signal-to-noise ratio of FTIR data by more than an order of magnitude over the levels of Fig. 2.

After reducing the $60-\mathrm{Hz}$ noise, a noise comparison was performed between the edge radiation source and a glower. Figure 3 shows transmittance data through the NicPlan microscope, where a $10-\mu \mathrm{m}$ pinhole was used at the sample stage while data was collected for one minute at $4 \mathrm{~cm}^{-1}$ resolution. The rms noise level is $0.62 \%$ transmittance (\%T) over $100 \mathrm{~cm}^{-1}$ for the edge compared to $12.5 \% \mathrm{~T}$ for the glower, a 20 -fold reduction. 


\section{APPLICATIONS}

Conventional IR microspectroscopy requires sample areas of $20 \mu \mathrm{m} \times 20 \mu \mathrm{m}$ or larger. The brightness of the Aladdin IR source permits a higher spatial resolution, as demonstrated by the following two examples.

To interpret the geological history of rocks, the fluid phase of the rocks may be analyzed. These fluids are commonly preserved as tiny $2-50 \mu \mathrm{m}$ fluid inclusions. The fluids can be a vapor, an aqueous or non-aqueous fluid, a silicate melt, or a combination. Inclusions exceeding $25 \mu \mathrm{m}$ in diameter are commonly studied using freeze/thaw techniques and visible microscopy; smaller inclusions must be studied spectroscopically.

Figure 4 shows infrared spectra of a $16-\mu \mathrm{m}$ inclusion that has a 6- $\mu \mathrm{m}$ "bubble" inside it. The upper spectrum shows the overall "liquid" phase. The peak at $2350 \mathrm{~cm}^{-1}$ is $\mathrm{CO}_{2}$, the broad peak around $3400 \mathrm{~cm}^{-1}$ is $\mathrm{H}_{2} \mathrm{O}$, and the small peaks below $3000 \mathrm{~cm}^{-1}$ are hydrocarbons. By changing the focus on the sample, the "vapor" phase in the bubble can be distinguished, as shown in the lower spectrum. This spectrum shows primarily $\mathrm{CO}_{2}$.

Hippocampus tissue of Alzheimer's disease patients is characterized by the buildup of plaques. Previous studies of these plaques have involved staining techniques that can contaminate and modify the protein structure. Other studies have extracted plaques for external investigation. Because protein structure is dependent on the material surrounding it, conclusions obtained from extracted plaques can be erroneous. With FTIR microspectroscopy, the tissues can be mapped to study plaques, neurofibrillary tangles and corpora amylacea in situ.

The upper trace in Figure 5 shows the spectrum of a corpus amylaceum smaller than $10 \mu \mathrm{m}$ in Alzheimer's white matter tissue. This is compared to the spectrum of the surrounding white matter. Note that the carbohydrate area around $1100 \mathrm{~cm}^{-1}$ is enhanced. The amide I profile

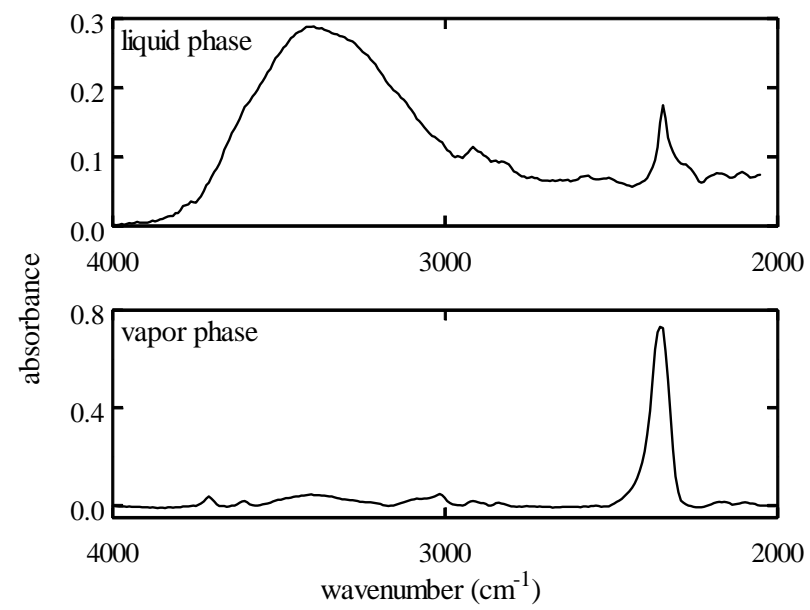

Figure 4. Measurement of a $16-\mu \mathrm{m}$ fluid inclusion with a $6-\mu \mathrm{m}$ vapor bubble. Measurement in transmission mode, $8 \mathrm{~cm}^{-1}$ resolution, 128 scans. Data courtesy of P. Brown and T. Lee, Dept. of Geology, University of Wisconsin-Madison.

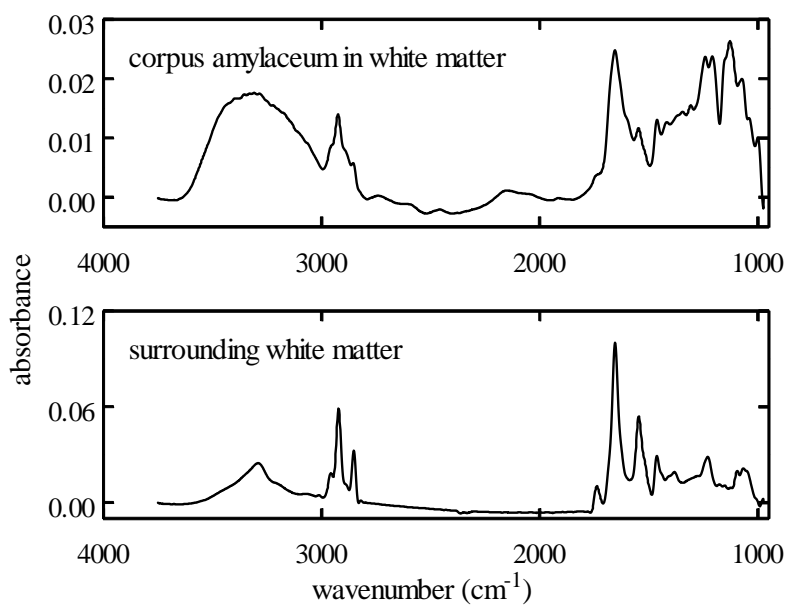

Figure 5. Measurement of Alzheimer's patient brain tissue showing the $<10 \mu \mathrm{m}$ corpus amylaceum and the white matter surrounding it, demonstrating different protein forms. Measurement in reflectance mode, 8 $\mathrm{cm}^{-1}$ resolution, 256 scans. Data courtesy of K. Gough and P. Bromberg, Dept. of Chemistry, University of Manitoba.

around $1650 \mathrm{~cm}^{-1}$ is quite different than for the white matter, indicating a change in the types of protein present. White matter is primarily ${ }^{\alpha}$-helical while a slight shift in the carbonyl frequency and appearance of shoulders at $1630 \mathrm{~cm}^{-1}$ and $1673 \mathrm{~cm}^{-1}$ indicates the presence of ${ }^{\beta}$-sheet or aggregated ${ }^{\beta}$-strands in the corpus amylaceum.

\section{ACKNOWLEDGMENTS}

The authors appreciate valuable discussions with $\mathrm{O}$. V. Chubar, M. A. Green, R. Reininger and W. S. Trzeciak. This work was supported by NSF grant DMR-95-31009.

\section{REFERENCES}

[1] M. A. Green, R. A. Bosch and W. S. Trzeciak, "Study of radiation along the extended axis of the long straight sections", in Synchrotron Radiation Center 1994 Yearbook (1994) p. 63.

[2] Daresbury 1984/5 Annual Report, Daresbury Laboratory, Warrington, Cheshire, UK (1985) front cover.

[3] O. V. Chubar and N. V. Smolyakov, J. Optics (Paris) 24, 117 (1993); Proc. 1993 IEEE PAC, 1626 (1993).

[4] R. Coïsson, Phys. Rev. A 20, 524 (1979).

[5] R. A. Bosch, M. A. Green and W. S. Trzeciak, "Incidental radiation along straight section axes in an electron storage ring", SRC Technical Note SRC-158, Synchrotron Radiation Center (1996).

[6] R. A. Bosch, Nucl. Instr. And Meth. A 386, 525 (1997).

[7] R. A. Bosch, T. E. May, R. Reininger and M. A. Green, Proc. SRI '95 (CD-ROM), Rev. Sci. Instrum. 67, 3346(K) (1996).

[8] D. Mossessian, "Infrared radiation from the Aladdin U3 straight section", SRC Technical Note SRC-171, Synchrotron Radiation Center (1996). 